${ }^{7}$ Department of Ophthalmology, Edouard Herriot Hospital, Lyon Cedex 03, France

E-mail: beby.francis@neuf.fr

Eye (2010) 24, 1293-1294; doi:10.1038/eye.2009.330; published online 15 January 2010

\section{Sir, Conjunctival lymphoma following treatment with cyclophosphamide}

Conjunctival lymphomas typically result from a monoclonal B-cell proliferation and represent either localized disease or a systemic malignancy. ${ }^{1}$ Most patients are over 60 years of age or are immunosuppressed. ${ }^{1}$ Patients typically present with insidious onset of an asymptomatic, salmon-coloured, well-demarcated mass. Uncommonly, lymphoma may occur following treatment with immunosuppressive medications. ${ }^{2}$ We present a case of conjunctival lymphoma that presented 4 years after treatment with cyclophosphamide. Although the association may be coincidental, our case emphasizes that physicians should be alert to the possibility of secondary malignancy occurring in the eye following systemic immunosuppression.

\section{Case report}

A 68-year-old man, with a history of sympathetic ophthalmia that occurred 6 years earlier, noticed a painless red mass in his right eye. Therapy for sympathetic ophthalmia included prednisone and a 6-month course of cyclophosphamide $50 \mathrm{mg} /$ day. His past ocular history was otherwise notable for multiple intraocular surgeries in the right eye for cataract and open-angle glaucoma. On presentation, his visual acuity was $20 / 25$ OU. Examination of the right conjunctiva revealed a salmon-coloured, vascularized mass from 1 o'clock to 3 o'clock (Figure 1). Fundus examination revealed a sunset glow appearance and chorioretinal scars bilaterally.

Biopsy revealed a diffuse lymphocytic stromal infiltrate (Figure 1b). Most lymphocytes were enlarged, with variable cytoplasm, irregular nuclei, and intranuclear inclusions (Dutcher bodies).

Immunophenotyping revealed strong CD20 and kappa light chain positivity. The histopathological diagnosis was a low-grade B-cell lymphoma that was mostly consistent with marginal zone lymphoma. This type of lymphoma is often referred to as mucosa-associated lymphoid tissue lymphoma. Treatment involves evaluation for systemic disease. Localized conjunctival lymphoma may be treated with radiotherapy, although some cases may be cured by local excision.

\section{Comment}

Long-term studies of patients treated with cyclophosphamide showed an increased risk for malignancy, including lymphomas. ${ }^{24}$ Hoffman et $a l^{2}$ observed a 2.4-fold overall increase in the incidence of malignancies, and an 11-fold greater incidence of systemic lymphomas in the general population. The risk appears to be dose-dependent, particularly with cumulative doses $>50 \mathrm{~g}$, but is also reported with lower doses, and may

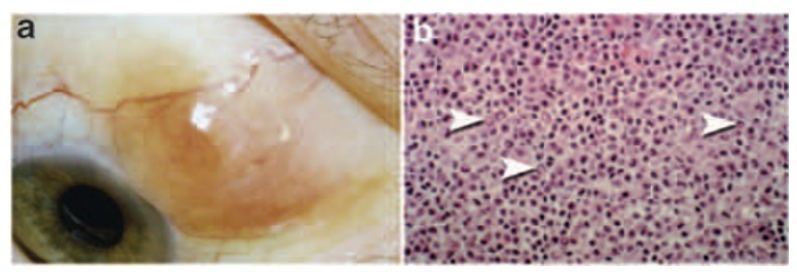

Figure 1 (a) Salmon-coloured, vascularized conjunctival mass of the right eye, extending from approximately 1 o'clock to 3 o'clock. Note the intact overlying conjunctiva and lack of feeder vessels. (b) Biopsy of the conjunctival mass, showing a nodular and diffuse lymphocytic infiltrate in the conjunctival stroma. Note the enlarged lymphocytes with variable cytoplasm and somewhat irregular nuclei. Higher magnification revealed intranuclear inclusions (Dutcher bodies, indicated by arrowheads). Most of the cells stained positively for CD20 and kappa light chains.

develop for up to 10 years. ${ }^{5}$ Our patient received a total of $9 \mathrm{~g}$. Potential mechanisms of malignancy include induced chromosomal alterations, altered immune-mediated anti-tumour surveillance, and increased susceptibility to oncogenic agents. ${ }^{3}$ Although the possibility remains that the association was spurious, our report of conjunctival lymphoma following treatment with systemic cyclophosphamide is the first report of such a secondary ocular malignancy, and illustrates that physicians should be alert to the possibility of secondary malignancy occurring in the eye following systemic immunosuppression.

\section{Conflict of interest}

The authors declare no conflict of interest.

\section{Acknowledgements}

This work was supported in part by The San Francisco Retina Foundation and The Pacific Vision Foundation.

\section{References}

1 Shields CL, Shields JA, Carvalho C, Rundle P, Smith AF. Conjunctival lymphoid tumors: clinical analysis of 117 cases and relationship to systemic lymphoma. Ophthalmology 2001; 108(5): 979-984.

2 Hoffman GS, Kerr GS, Leavitt RY, Hallahan CW, Lebovics RS, Travis WD et al. Wegener granulomatosis: an analysis of 158 patients. Ann Intern Med 1992; 116(6): 488-498.

3 Radis CD, Kahl LE, Baker GL, Wasko MC, Cash JM, Gallatin A et al. Effects of cyclophosphamide on the development of malignancy and on long-term survival of patients with rheumatoid arthritis. A 20-year followup study. Arthritis Rheum 1995; 38(8): 1120-1127.

4 Kempen JH, Gangaputra S, Daniel E, Levy-Clarke GA, Nussenblatt RB, Rosenbaum JT et al. Long-term risk of malignancy among patients treated with immunosuppressive agents for ocular inflammation: a critical assessment of the evidence. Am J Ophthalmol 2008; 146: 802-12.e1. Jun 23.

5 Travis LB, Curtis RE, Glimelius B, Holowaty EJ, Van Leeuwen FE, Lynch $\mathrm{CF}$ et al. Bladder and kidney cancer following cyclophosphamide therapy for non-Hodgkin's lymphoma. J Natl Cancer Inst 1995; 87(7): 524-530. 


\author{
NJS London ${ }^{1}$, C Siverio $\mathrm{Jr}^{2}$ and ET Cunningham $\mathrm{Jr}^{1,3}$ \\ ${ }^{1}$ California Pacific Medical Center, San Francisco, \\ CA, USA \\ ${ }^{2}$ The Instituto Nacional de Oftalmologia, Lima, Peru \\ ${ }^{3}$ Department of Ophthalmology, Stanford \\ University School of Medicine, Stanford, CA, USA \\ E-mail: emmett_cunningham@yahoo.com
}

Eye (2010) 24, 1294-1295; doi:10.1038/eye.2009.333; published online 15 January 2010

\section{Sir, \\ Coats' disease associated with muscular dystrophy treated with ranibizumab}

Coats' disease can be associated with muscular dystrophy. We present a case in which ranibizumab was used for Coats' disease associated with a muscular dystrophy.

\section{Case report}

An 18-year-old man came to our department because he had noted decreased vision in his left eye. The patient had no systemic complaints. The best-corrected visual acuity (VA) was 20/20 in his right eye (RE) and count fingers in his left eye (LE). The anterior segment was unremarkable. Fundus examination showed hard circinate exudates, telangiectatic vessels, microaneurysms, and venous dilatation only in the LE (Figures 1a and b). The patient underwent fluorescein angiography (Figures $1 \mathrm{c}$ and d) and optic coherence tomography (OCT) (Figure 2). Coats' disease was confirmed.

A complete blood count, liver and muscle enzymes and coagulation testing was performed. The patient showed a consistently elevated creatine phosphokinase on repeat testing of $3000 \mathrm{UI} / \mathrm{ml}$. We referred the patient to a neurologist, who performed the diagnosis of facioscapulohumeral dystrophy (FSHD).

Peripheral argon laser photocoagulation and three ranibizumab injections were administered to the LE. The VA improved to 20/60 and has stayed stable with 1 year of follow-up (Figure 3 ).

\section{Comment}

Coats' disease is characterized by vascular abnormalities, commonly telangiectatic vessels and retinal exudation. Normally only one eye is involved and predominantly men are affected before 20 years of age ${ }^{1,2}$ Usually, it is not necessary to do a systemic study with typical signs of Coats' disease but there are some cases of muscular and facioscapulohumeral dystrophies associated with Coats'

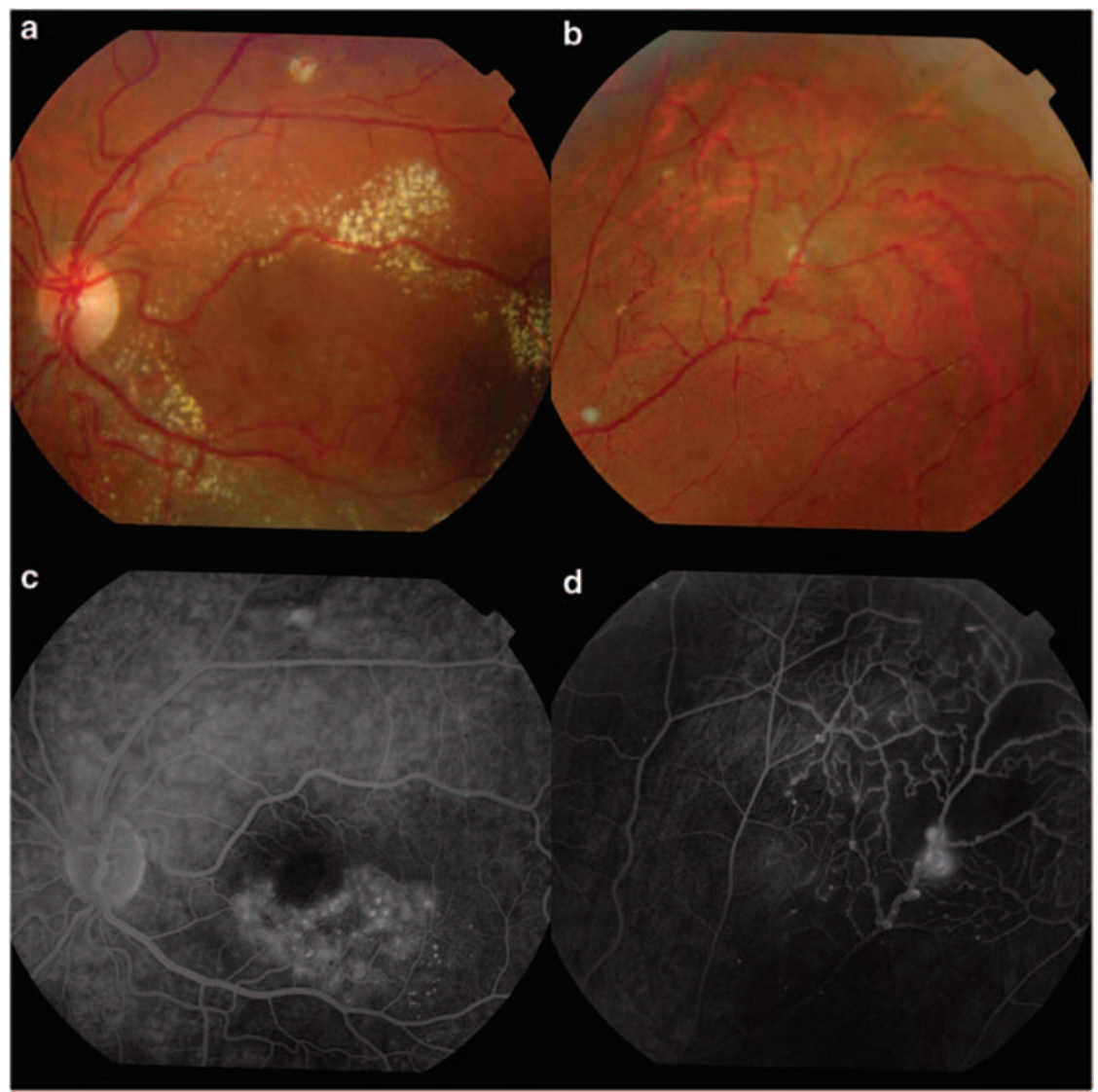

Figure 1 (a) Funduscopy of left eye with the presence of hard circinate exudates. (b) Funduscopy of periphery of left eye in which telangiectatic vessels, venous dilatations, microaneurysms can be observed. (c) Angiogram of left eye showing exudation in posterior pole. (d) Angiogram of temporal periphery of left eye showing telangiectasia and tortuosity of retinal vessels. 